\title{
Fachlich-biographische Notizen zur publizistischen Tätigkeit katholischer Geistlicher in der deutschen Presse (19. Jh.)
}

Eine allgemeine Einführung in die fachlich-biographischen Notizen findet der Leser in CS Nr. 1/1968, S. 74-76. Zu wiederholen wäre der Hinweis, daß für die Auswahl der erwähnten Personen ihre Aktivitäten in der Publizistik, also vornehmlich in Zeitungen und Zeitschriften, wichtig waren. Die meisten Angaben sind notgedrungen aus Sekundärquellen gewonnen: Fehler dieser Fundstellen werden also, solange keine Kontrollstelle belegt werden oder zu den Primärquellen vorangeschritten werden kann, zunächst einmal übernommen: Daher auch die genauen Belege mit Chiffren, deren Auflösung auf der dritten Umschlagseite zu finden ist. Alle Hinweise und Korrekturen sind der Redaktion sehr willkommen.

\section{Folge}

Niedermayer, Andreas, Gründer, Herausgeber, - 11. 10. 1835 Niederviehbach in Niederbayern, + 17. 1. 1872 Frankfurt, 1858 Priester, Gründer und Herausgeber der Zeitschrift Die katholische Bewegung 1868-1870, Sekretär im Zentralkomitee der Katholiken Deutschlands. KK, 3254. - Gründer der Katholischen Bewegung 1866. 9, 46, vgl. $6,25,18,242$ und 7 II, 307. - Mitbegründer des Frankfurter Volksblatles. 6, 50.

Niegler, Georg, Theologe in Bamberg, Herausgeber des Religionsfreund fïr Katholiken 1828. 9, 22.

Niesen, Johann Adam, Redakteur, * 1833 Mönchen-Gladbach, 1878 Pfarrer in Elsig bei Euskirchen, 1888 in Aldenhoven, Redakteur des Euskirchener Volksblattes. 6,96 .

Nikel, Johann, Herausgeber, * 18. 10. 1863 Schrau in Oberschlesien, +28.6 . 1924 Breslau, 1886 Priester, 1900 Professor, 1914 Domherr, Mitherausgeber der Biblischen Zeittragen seit 1908. KK, 3268 .

Nisius, SJ, Johann Baptist, Chefredakteur, * 26. 5. 1853 Trier, t 6. 11. 1922 Innsbruck, 1879 Jesuit, 1888 Professor in Innsbruck, 1898 Schriftleiter der Zeitsehrift filr katholisehe Theologie. KK, 3272 .

Noldin, SJ, Hieronymos, Chefredakteur, *30. 1. 1838 Salurn/Tirol, t 7. 11. 1922 Wien, 1865 Jesuit, 1885 Professor in Innsbruck, Schriftleiter der Zeltschrift für katholische Theologie $1886-90$. KK, 3280 .

Noppel, SJ, Konstantin, Mitarbeiter, *2. 8. 1883 Radolfzell in Baden, Dr. phil. et theol., 1909 Jesuit, seit 1911 Mitarbeiter der Stimmen der Zeit, 1932 Rektor des Collegium Germanicum in Rom und Professor. KK, 3282.
Nostadt, Jakob, Ilerausgeber und Chefredakteur - 1841 Ebersheim bei Mainz, + 19. 1. 1911 Wies baden, Pfarrer in Büdesheim und Sozialreformer Redlakteur des Mainzer Journal. 6, 47, - Seit 1. 10. 1878 Herausgeber der Römischen Korrespondenz. 18,9 , vgl. 9,61 . - Chefredakteur des Mainzer Journal 1874-85. 6, 46. - Chefredakteur der Vergangenheit und Gegenwart. 19, 16.

Notstadt, Joseph, * 1826 Bensheim, † 1901, 1850 Priester, 1885 Domkapitular in Mainz, Redakteur des Katholischen Volkshlattes, Mitglied des Augustinus-Vereins seit der Gründung. 6, 47.

Nostitz-Rieneck, SJ, Robert Graf von, Redakteur und Mitarbeiter, "18. 3. 1856 Schloß Slabez/Böhmen, $t$ 16. 7. 1929 Freising, 1881 Priester, 1891 Professor in Feldkirch, Redakteur der Stimmen der Zeit in München, 1922 Spiritual in Freising, Mitarbeiter der Stimmen der Zeit und historischer Zeitschriften. $\mathrm{KK}, 3286$.

Nüdling, Ludwig, Herausgeber, * 26. 2. 1874 Poppenhausen, 1897 Priester, Herausgeber des Kommunionglöckleins, Jugendschriftsteller. KK, 3291 .

Oberdörfer, Karl, Gründer, * 31, 10. 1860 Ahlefeld/Sieg, 1888 Priester, 1906 Professor, 1924 Domkapitular, Diözesanpräses der Borromăus-Vereine Mitbegründer der Monatsblätter für den katholischen Religionsunterricht. KK, 3299 .

Oberdörfer, J. Peter, Redakteur und Mitarbeiter, * $1852 \mathrm{Breidt} / \mathrm{Sieg}$ + 28. 2. $1925 \mathrm{Köln}, 1875$ Priester, 1882-85 Redakteur der Tremonia, seit 1886 Kaplan und Mitarbeiter des Rheinischen Merkur, Sozialpolitiker, Gründer von Arbeitervereinen, Mitglied des Áugustinus-Vereins. 6, 54; 9, 64.

Oberthür, Franz, Mitarbeiter, * 6. 8. 1745 Würzburg, $t$ 30. 8. 1831 ebenda, 1769 Priester, 1773 
Professor, 1821 Domkapitular. KK, 3311. - Mitarbeiter der Zeitschrift Von der kathollschen Kirche. 13, 202. - Mitarbeiter des Religionsfreund Itir Katholiken. 13, 145. - Mitarbeiter des Literarischen Magazin für Kathollken und deren Freunde. 9, 18.

Oechsler, Hermann, Mitarbeiter, * 18. 2. 1849 Ettlingen, +5.3 . 1930 Ebringen bei Freiburg 1874 Priester, Schriftsteller, Ehrendoktor der Theologischen Fakultät Freiburg 1929, Mitarbeiter am Freiburger Diözesanarchiv. KK, 3319.

Orsbach, Martin von, * 1777 , +1846 , Stiftsherr des Aachener Mūnsters, Mitarbeiter des Nathanael. 13,107 und 221 .

Ortileb, Eduard, Herausgeber, * 16. 7. 1807 Oberndorf am Neckar, + Jan. 1861 Cannstatt, 1834 Priester, Kirchenmusiker, Herausgeber des Organs für kirchliche Tonkunst $1852-57$. $\mathrm{KK}, 3368$.

Oswald, Johann Heinrich, Mitarbeiter, * 6. 6. 1817 Dorsten, + 7. 8. 1903 Braunsberg, 1840 Priester, 1846 Professor in Paderborn, 1875 in Braunsberg. KK, 3379. - Mitarbeiter des Christkatholischen Magazins zur Belehrung und Erbauung für alle Stände. 13, 214.

otte, Waldemar, Chefredakteur und Mitarbeiter, -29. 3. 1879 Leuber in Oberschlesien, Pfarrer in Liegnitz, Kirchenhistoriker, Chefredakteur der Neisser Zeitung 1919-21. KK 33, 84

Otten, Alois, Gründer, * 9. 6. 1853 Paderborn, + 9. 5. 1900 ebenda, 1876 Priester, 1889 Professor in Paderborn und 1889 mit Heiner Grander der Zeitschrift Der katholische Seeisorge. KK, 3384, vgl. KU, 578 .

Overhage, Heinrich, Mitarbeiter, * 1. 12. 1806 Ahlen, + 23. 11. 1873 Werne, 1831 Priester, 1848 Dechant Volksschriftsteller. KK, 3394 - Mitarbeiter des Hausbuch fïr christliche Unterhaltung. $9,48$.

Overmans, SJ, Jakob, Redakteur und Mitarbeiter. * 26. 1. 1874 Breyell, 1892 Jesuit, Dr. phil. et theol., seit 1910 Mitarbeiter der Stimmen der Zeit, 1924 Professor in Tokio, 1929 Redakteur der Stimmen der Zeit und Professor in Frankfurt. KK, 3394 .

Pachtler, SJ, Georg Michael, (Ps. Annuaris Ossegg), * 14 . 9. 1825 Bad Mergentheim, + 12.8. 1889 Exaeten/Holland, 1848 Priester, 1864 Professor in Feldkirch, 1871 Chefredakteur der Stimmen aus Maria Laach. KK, 3406 .

Pangerl, SJ, Franz Sales, Redakteur, * 23. 1. 1879 Aigen/Nicder-Osterreich, 1897 Jesuit, Professor in Innsbruck, Redakteur der Zeltschrift für katholiselue Theologie. KK. 3414

Parmet. Adalbert, Redakteur, * 30. 7. 1830 Mūnster, $+1898,1853$ Priester, 1869 Professor in Münster, Redakteur des Münsterschen Sonntagsblattes. $\mathrm{KK}, 3426$.

Parsch, Pius, Herausgeber, * 18. 5. 1884 Neustift in Mähren 1909 Priester, Professor in Klosterneuburg, Herausgeber der Zeitschrift Der Seelsorger. $\mathrm{KK}, 3427$

Passy, Anton (Ps. Passi), Mitarbeiter, * 31. 3. 1788 Wien, + 11. 3. $1847^{\prime}$ ebenda, 1820 Redemptorist, Bibliothekar, Kirchenhistoriker und Erbauungsschriftsteller. KK, 3432. - Mitarbeiter des Allgemeinen Kirchen- und Religionsfreundes. 13, 200. - Mitarbeiter des Chrysostomus. 13, 207. Pauli, Geistlicher in Fulda, Redakteur der Fuldaer Zeitung, 19,82 .
Pell, Georg, Gründer und Redakteur, *4. 11. 1849 Passau, $f$ 19. 1. 1927 ebenda, 1874 Priester, 1888 Professor und Rektor in Passau, 1891 Mitbegründer und Redakteur der Theologiseh.Praktischen Monatsschrift. KK, 3463 , vgl. KÛ, 1081.

Pesch, SJ, Tilmann (Ps. Gottlieb), Grũnder, Redakteur und Mitarbeiter, * 1. 2. 1836 Köln, † 18. 10. 1899 Valkenburg/Holland, 1866 Priester, 1867 Professor in Maria-Laach, 1872 Schriftleiter der Stimmen aus Maria Laach in Tervueren (Belgien), Gründer der Ergänzungshefte zu den Stimmen aus Maria Laach, Mitarbeiter der Germania. KK, 3488. - Einer der ersten Mitarbeiter der Stimmen aus Maria-Laach. 18. 53

Peschke, Kurat an St. Anton in Breslau, Herausgeber und Redakteur des Apologet 1854-47 in Breslau. 13, 222 .

Peters, Franz Joseph, Chefredakteur, * 10. 4. 1875 Bonn, 1897 Priester, 1913 Professor, 1914-21 Chefredakteur der Kölner Pastoralblätter. KK, 3497.

Pfaff, Johann Leonhard, Mitarbeiter, *18. 8. 1775 Hünfeld/Hessen, † 3. 1.'1848 Fulda, 1798 Priester, 1802 Professor, 1831 Bisch of von Fulda. Mitarbeiter des Mainzer Sonntagsblattes und der Katholischen Literaturzeitung. A XXV, 594. - Mitarbeiter der Rheinischen Volkshalle. 1 II, 419. - Mitarbeiter der Katholischen Kirchenzeitung. 9, 22.

Pfeneberger, Josef, Chefredakteur und Mitarbeiter, * 4. 8. 1880 Netternbach/Oberösterreich, 1903 Priester, 1906-09 Chefredakteur des Linzer Volksblattes, 1925 Landtagsabgeordneter und 1927 Landesrat. KK, 3535 .

Pfister, Adolf, Chefredakteur und Redakteur, " 26 9. 1810 Hechingen, + 29. 4. 1878 Oberdischingen in Württemberg, 1833 Priester, Chefredakteur des Katholischen Schuiwochenblattes 1860 und seit 1861 zusammen unit Rolfus Redakteur des Süddeutschen Katholisehen Schulwochenblattes. KK, 3537 .

Pflanz, Benedikt Alois, Herausgeber, 25. 11. 1797 Espacliweiler bei Ellwangen, $t$ 23. 11. 1844 Schörzingen, 1820 Priesterweihe, 1828 Professor. 1831 Landtagsabgeordneter, Herausgeber der Freimiitigen Blătter über Theologie und Kirchentum 1830-44. IK, 3540 , vgl. 1 I, 244. - IHerausgeber der Sonntagsfreude für die christiliche Jugend. 9,48 .

Pfleger, Karl, * 8. 10. 1883 Dachstein/Elsaß, Pfarrer, Mitarbeiter des Hochland und der Seele. KK, 3542

Pflügl, Albert Edler von, Gründer und Mitarbeiter, * 1818 Linz, + 5. 3. 1886 Peuerbach, 1841 Priester, 1865 Landtagsabgeordneter, 1873 Reichstagsabgeordneter. Gründer des Volksblattes für Religion und Gesetz. KK, 3544.

Pfülf, SJ, Otto, Redakteur, * 28. 1. 1856 Speyer, 1875 Jesuit, Professor in Ditton-Hall, Redakteur der Stimmen aus Maria Laach, Spiritual. KK 3550 .

Pidoll, Domdekan, 1839-41 Mitarbeiter an den Deutselien Blättern fïr Protestanten und Katholiken. 1 I, 239

Pieper, Anton, Herausgeber, * 20. 3. 1854 Lüdinghausen/Westf., + 24, 12. 1908 Münster, Professor für Kirchengeschichte in Münster. KK, 3577. - Mitherausgeber der Zeitschrift für Vaterländische Geschichte und Altertumskunde. KU, 1235. 
Pleper, August, Herausgeber, * 14. 3. 1866 Eversberg im Sauerland, Dr. phil. et. theol., 1889 Priester, 1903-19 Generaldirektor des Volksvereins für das katholische Deutschland, Mitglied des Landtages und des Reichstages, seit 1901 Herausgeber der Präsides-Korrespondenz, die seit 1922 als Fuhrer-Korrespondenz erscheint. KK, 3577. - Mitglied des Augustinus-Vereins seit Grūndung, sozialpolitischer Schriftsteller. 6, 92.

Pleper, Karl, Herausgeber, * 14. 11. 1877 Dortmund, 1902 Priester, 1928 Professor in Paderborn, 1921-24 Herausgeber der Zejtschrift für Missionswissenschaft, seit 1929 Mitherausgeber der Zeitschrift Theologie und Glaube. KK, 3578, vgl. 9, 97.

Pletz, Joseph, Gründer und Herausgeber, * 3. 1. 1788 Wien, $t$ 30. 3. 1840 ebenda, 1812 Priester, Professor, 1827 Domherr, 1836 Hofpfarrer, gründete 1828 die Neue Theologlsche Zeitschrift. KK 3619. - Herausgeber der Neuen Theologischen Zeitschrift 1813-25. 1 I, 243.

Pöllann, Ansgar, Herausgeber und Redakteur 21. 9. 1871 Hechingen, $t$ 24. 6. 1933 Eltville 1893 Benediktiner, 1903 Herausgeber der Gotlesminne, Schriftleiter am Bayrischen Kurier, Feldgeistlicher und Spiritual in Johannisberg. KK, 3628. - Herausgeber der Gottesminne 1903-17. $9,93$.

Pösl, Friedrich von, Herausgeber und Chefredak teur, * 1. 9. 1806 Landshut, + 27. 7. 1876 Puchheim/Osterreich, 1829 Priesterweihe, $1835 \mathrm{Re}$ demptorist, 1848 in USA, Herausgeber der Passauer Blätter zur Erbauung und Belehrung 1836 bis 1843. KK, 3633. - Redakteur der Blatter zur Belehrung und Erbauung. 13, 207.

Poggel, Heinrich, Herausgeber und Mitarbeiter, -12. 10. 1858 Paderborn, + 3. 8. 1928 ebenda, 1888 Priesterweihe, Mitherausgeber von Theologle und Glaube seit 1909, Mitarbeiter vieler Zeitschriften. $\mathrm{KK}, 3637$.

Pohl, Julius, Gründer, Redakteur und Mitarbeiter, 13. 6. 1830 Frauenburg/Ostpreußen, + 9. 3. 1909 Norbertusheim/Oberzell bei Würzburg, 1857 Priester, 1863 Domvikar, Mitbegründer der Ermiändischen Zeltung und Redakteur bis 1877 . KK, 3640 . - 1872 mit Kolberg Gründer der Ermländischen Zeitung. KU, 351 .

Ponholzer, Bartholomăus, Chefredakteur, *12. 3. 1827 Ostersee/Bayern, $f$ 25. 4. 1892 Áugsburg, 1853 Priesterweihe, Diōzesanpråses. KK, 3651. Chefredakteur des Wochenblaties fur das ehristllehe Volk. 18, 211 .

Praxmarer, Johann Nepomuk Anton, Grônder, Chefredakteur und Mitarbeiter, " 16. 5. 1853 Bingen, $t$ 13. 11. 1934 Aschaffenburg, 1879 Priester, 1880 Chefredakteur des Ambrosius, $1886 \mathrm{Re}$ dakteur der Katholischen Bewegung, gleichzeitig Chefredakteur der Sozialpolitischen Konferenz iür den Mittelrhein, 1893 Gründer und Chefredakteur des Stern der Jugend. KK, 3577, vgl. KÜ, 48. Mitarbeiter der Deutsehen Katholikenzeltung 1920-21. 9, 94.

Raes, Andreas, Grônder und Mitarbeiter, * 6.4. 1794 Sigolsheim, OberelsaB, + 17. 11. 1887 StraBburg, 1816 Priester, 1825 Professor in Mainz, dann in Straflburg, 1842 Bischof von Mainz, 1821 Mitbegründer des Kathollk. KK, 3764, vgl. 6, 5. - Mitarbeiter des Allgemeinen Religlons. und Klrchenfreund. 13, 200. - Mitarbeiter der Katholischen Kirchenzeltung. 13, 203.

Ralch, Johann Michael (Pseudonym Otto Beuren), Herausgeber und Chefredakteur, * 17. 1. 1832
Ottubeuren, + 28. 3. 1907 Mainz, 1858 Priester, 1859 Sekretăr des Bischofs Ketteler, 1900 Domdekan in Mainz, Herausgeber des Katholik und Leiter der Frankfurter zeitgemäBen Broschüren seit 1887. KK, 3771, vgl. KU, 390. - Von 1890 bis 1907 Herausgeber des Katholik. 1 I, 196.

Rampf, Michael von, Gründer, * 4. 10. 1825 Mūnchen, t 29. 3. 1901 Passau, 1848 Priesterweilie, 1864 Domkapitular in München, 1889 Bischof von Passau. KK, 3782. - 1891 Mitbegründer der Theolodlsch-Praktischen Monatsschrift. KÜ, 1081.

Ratzinger, Georg, Gründer, Redakteur und Mitarbeiter, * 3. 4. 1844 Rickering in Niederbayern, + 3. 12. 1899 München, 1867 Priester, seit 1875 Mitglied des Deutschen Reichstages und des Bayrischen Landtages, einer der bedeutendsten katholischen Sozialreformer. KK, 3802. - Korrespondent der Deutschen Reichszeitung in München, seit 1869 Mitarbeiter der Historisch-Politischen Blätter, am Bayrischen Vaterland und an der Germania sowie an der Passauer Donauzeltung und der Augsburger Postzeitung. Redakteur des Fränkischen Volksboten $1870 / 71$ in Würzburg und 1873-83 Redakteur des von ihm begründeten Volksfreund in München. A LIII, 215 f. - Mitarbeiter des Chilianeum. 9, 44.

Rebbert, Josef, Gründer, Verleger, Herausgeber und Redakteur, *19.5. 1837 Winterberg in Westfalen, $t$ 13. 7. 1897 Paderborn, 1860 Priester, 1871 Professor in Paderborn, 1878 Gründer und Schriftleiter des Sonntagsblattes Leo. KK, 3819, vgl, KU, 666. - Verleger des Leo in Paderborn. 18, 627, vgl, 6, 48. - Herausgeber der Paderborner Blătter für Kirchliche Wissenschaft und Praxls. 9, 71.

Rechbercer, Augustin, Gründer, * 18. 11. 1800 Linz, † 7./8. 12. 1864 ebenda, 1828 Prolessor tür Dogmatik in Linz, 1859 Domherr, Gründer der Linzer Theologisch-Praktlschen Quartalschrift 1852. KK, 3824 .

Relchmann, SJ, Matthias, Mitarbeiter, * 24.2. 1852 Mühlhausen in Württemberg, +8.8 . 1936 Pullach bei München, 1869 Jesuit, seit 1883 Mitarbeiter der Stimmen der Zelt, Kirchenhistoriker. $\mathrm{KK}, 3856$.

Reinarz, Dekan in Krefeld, Mitbegründer der Rhelnischen Volkshalle. 1 II, 8. - Mitbegründer der Deutschen Volkshalle. 1 II, 127.

Relnfried, Karl, Mitarbeiter, *25. 4. 1842 Būhl in Baden, + 5. 10. 1917 Moos beí Bühl, 1867 Priester, Pfarrer in Moos, seit 1877 Mitarbeiter des Frelburger Diözesanarchlvs und mehrerer katholischer Tageszeitungen. KK, 3872 .

Reinke, Lorenz, Mitarbeiter, * 6. 2. 1797 Langförden in Oldenburg, $t$ 4. 6. 1879 Münster. 1837 Professor für alttest. Exegese in Münster, Ehrendoktor der Theologie und Philosophie, seit 1852 Domkapitular daselbst. KK, 3879. - Mitarbeiter des Christkatholischen Magazins zur Belehrung und Erbauung für alle Stände 1841-48. 13, 214.

Relter, Josef, Chefredakteur, * 1805 St. Florian in Oberōsterreich, +22.4 . $1876 \mathrm{Linz}$, Professor, Gründer des Bonifatiusvereins, Chorherr in St. Florian, Chefredakteur der Linzer TheologischPraktischen Quartalschrift und der Katholisehen Blätter in Linz. KK, 3894.

Relthmayr, Franz Xaver, Herausgeber und Mitarbeiter, 16. 3. 1809 Illkofen bei Regensburg, † 26. 1. 1872 München, 1839 Professor für neutestamentl. Exegese in München. KK, 3897. - Mitarbeiter der Neuen Slon. 13, 244. - Mitherausgeber des Archlvs fur Theologlsche Llteratur. 1 I, 244, vgl, 9, 26. 